\title{
The wavelet transforms technique in the com- puter-aided system for controlling the quarry unmanned vehicles
}

\author{
Ivan Chicherin ${ }^{1 *}$, Boris Fedosenkov ${ }^{1}$, Dmitriy Dubinkin ${ }^{2}$, and Wang Zhenbo ${ }^{3}$ \\ ${ }^{1}$ T.F. Gorbachev Kuzbass State Technical University, Department of Information and Computer- \\ aided Manufacturing Systems, 650000 Kemerovo, 28 Vesennyaya st., Russian Federation \\ 2 T.F. Gorbachev Kuzbass State Technical University, Department of Metal-Cutting Machines and \\ Tools, 650000 Kemerovo, 28 Vesennyaya st., Russian Federation \\ ${ }^{3}$ Shenyang University of Technology, Shenyang Mechanical and Electrical Engineering College, \\ 110870 Shenyang, Liaoning, No. 111 West Shenliao Road, Economic \& Technical Development \\ Zone, People's Republic of China
}

\begin{abstract}
Introduction. Purpose of the work. Within the framework of the computer-aided system, a technology has been formed for the method of controlling the current trajectories (CTs) of unmanned vehicles (UMVs) when they move along routes in a quarry in open pit mining. The purpose of the presented studies is to analyze the application of a wavelet transforms technique to the problem of routing unmanned vehicles when they move along routes within open pit roads. Methodology. The results of modeling certain one-dimensional signals corresponding to the UMV current trajectories when they deviate to the left / right from a nominal axial trajectory (NAT), as well as their time-frequency representations in a wavelet medium are presented. An algorithm of the procedure for displaying scalar UMV CT control signals in a complex medium of timefrequency wavelet transforms has been developed and described. Such a transformation allows for a functionally transparent and informationcapacious monitoring of the UMV movement and efficiently manage the processes of trajectory routing dump trucks in an open pit. Research results, analysis. The processes of modifying the UMV movement current trajectories under the control of the computer-aided system are generated using wavelet transforms methods. They are based on algorithms for projecting the trajectory signals with a time-dependent frequency (chirp signals) onto a set of wavelet functions as part of a wavelet thesaurus (wavelet dictionary), executing certain wavelet matching pursuit procedures, and displaying the CT scalar signals in a specific multidimensional medium of Cohen's class time-frequency distributions. The simulation results in the form of the current trajectory (CT-) signals waveforms and their threedimensional time-frequency representations as Wigner maps showing the UMV movement in a start-stop mode, as well as the signals of formed continuous deviation trajectories when they leave to the left and to the right from the NAT, are presented. An algorithm for the formation of 3D-
\end{abstract}

\footnotetext{
*Corresponding author: chiv.iap@kuzstu.ru
} 
representations of UMV current trajectory one-dimensional signals is presented. Conclusion. The conclusion is made that the mathematical technique of wavelet transforms is the most expedient and effective means for computer-aided monitoring and controlling the dynamics of UMV movement along routes within open pit roads.

\section{Introduction, purpose of the work}

In open pit mining, within the framework of the «Smart Quarry» global structure [1], rock and minerals transferring from a working bench face to an unloading point is carried out by heavy-duty dump trucks on an unmanned basis - by autonomous heavy platforms [2], or, in other words, unmanned vehicles (UMVs). The article presents the structure and description of the wavelet transforms technique, with the help of which, within the framework of the computer-aided system, the technology for controlling the current trajectories of UMVs when they move along the routes in an open pit in the conditions of opencast mining is formed.

\section{Methodology}

It is shown in the publication [3] that for computer-aided monitoring and controlling the dynamics of the UMV movement along the routes within quarry roads, the most expedient and effective mathematical tool, which makes it possible in a semantically transparent and functionally capacious form to realize real-time looking after the operating UMVs, is a wavelet transforms technique.

This paper presents the results of modeling one-dimensional signals, as well as their time-frequency representations in a wavelet medium, corresponding to the current trajectories when they deviate to the left / right from the nominal axial trajectory (NAT) during the UMV movement along the quarry routes.

In addition, an algorithm for the procedure for displaying scalar signals of controlling the UMV current trajectories (CT) in a complex medium of time-frequency wavelet transforms has been developed and described. Such a transformation allows for a functionally transparent and information-capacious monitoring of the UMV movement and efficiently controlling the processes of trajectory routing of dump trucks in the quarry.

\section{Research results, analysis}

The processes of modifying the UMV current trajectories [1] controlled by the computeraided system are generated using the methods of wavelet transforms. They are based on algorithms for projecting trajectory signals with a time-dependent frequency (chirp signals) $[1,4]$ onto a set of wavelet functions as part of a wavelet thesaurus (wavelet dictionary) [5], wavelet matching pursuit procedures $[4,5]$, and representing the scalar signals in a specific multidimensional medium of Cohen's class time-frequency distributions. [4, 6, 7-10].

In particular, when passing on a route with an oncoming unmanned vehicle or when carefully bypassing or avoiding any obstacle, the UMV under the commands of the computer-aided dispatching system, switches to the start-stop mode of movement. The corresponding example is shown in Fig. 1 a) with a pulse waveform of the signal for the start-stop mode of the UMV movement along the CT going to the left of the NAT (that is, 
the corresponding chirp signal [4], a signal with a time-dependent instantaneous frequency), which can be written analytically as follows:

$$
\begin{gathered}
x(t)=\frac{A_{0}}{2}+\sum_{k=1}^{10}\left(A_{k} \cdot \cos \left(\frac{\omega_{k}}{t}\right)+B_{k} \cdot \sin \left(\frac{\omega_{k}}{t}\right)\right) \\
A_{0}=\frac{2 x_{d_{\max }}}{\lambda} ; A_{k}=\frac{x_{d_{\max }}}{k \cdot \pi}\left(\sin \left(\frac{2 \cdot k \cdot \pi}{\lambda}\right)\right) ; B_{k}=\frac{x_{d_{\max }}}{k \cdot \pi}\left(\sin \left(\frac{k \cdot \pi}{\lambda}\right)\right)^{2} ; \omega_{k}=\frac{2 \cdot \pi \cdot k}{T}
\end{gathered}
$$

After decomposing the signal according to the wavelet matching pursuit algorithm (WMP-algorithm) [5, 4], we obtain its time-frequency distribution - TFD [4, 7, 9] (Fig. 1 c), and the latter adequacy is established by comparing the original signal (Fig. 1 a) with the one reconstructed from spectral components (Fig. $1 \mathrm{~b}$ ).

The Wigner map $[4,8,9,11,12]$ clearly shows the exponentially falling character of the chirp signal frequency for the start-stop mode, and, consequently, the discrete trajectory which deviates to the left of the NAT. In particular, we see that the initial signal of the UMV start-stop movement mode (Fig. 1 a), as well as the signal reconstructed according to the UPN algorithm (Fig. 1 b) are displayed on the wavelet map (W-map) by seven timefrequency atoms $(\mathrm{HF})[3,6]$. Here, the first five pulses are displayed with five TFAs on the Wigner time-frequency distribution, the sixth - with four TFAs. The rest feeble, low power, TFAs correspond to forward and rear pulse fronts, as well as to some computation interference and noise.

a)

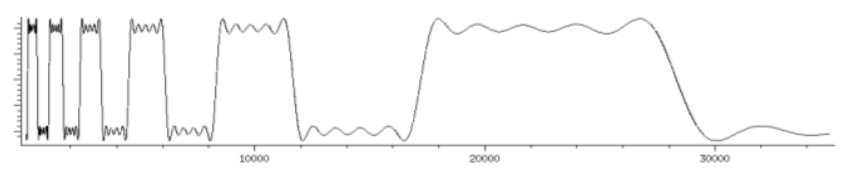

b)

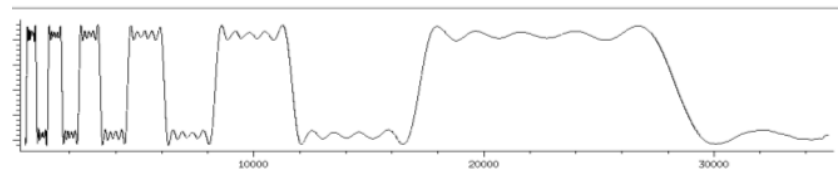

c)

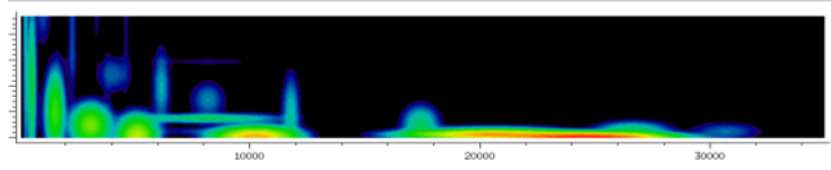

Fig. 1. The waveform of the signal corresponding to the start-stop mode of UMV moving along the CT leaving to the left of the NAT, and its time-frequency distribution

Fig. 2 shows the signal of the forward transient process when the CT deviates to the left of the NAT (in a centered form); in addition, the signal of this mode, reconstructed by the WMP-algorithm, as well as the signal approximation error are also presented here. In this case, the process takes the form of a chirp signal with a falling time-dependent instantaneous frequency. 
The figure also shows the Wigner map for the forward transient process with CT deviating to the left of the NAT, experimentally recorded by the hardware and software complex in the directories of the external and autonomous control subsystems (ECSS and ACSS). The presented Wigner map demonstrates a chirp signal with a decrease in the instantaneous frequency from $0.5 \mathrm{~Hz}\left(\omega=3.14 \mathrm{~s}^{-1}\right)$ to $f=0.02 \mathrm{~Hz}\left(\omega=0.13 \mathrm{~s}^{-1}\right)$. Such a deviation of the UMV CT can be caused by the presence on the route of a dynamic obstacle in the form of a group of walking people, a stationary obstacle such as temporarily installed lighting facilities, electrical switchgear boxes with air or cable connection, or oversized pieces of rock mass lying on the roadbed.
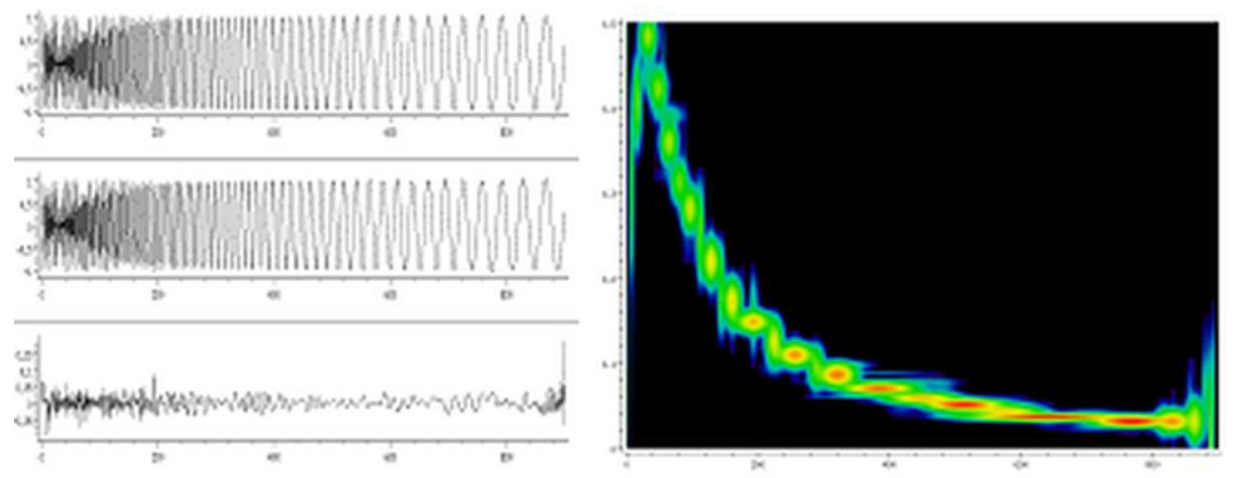

Fig. 2. A one-dimensional signal (in the UMV CT external and autonomous control subsystems) of forming the CT deviating to the left from the NAT, and its Wigner map (W-map)

Fig. 3 shows the signal in the centered form of the forward transient process when the CT deviates to the right of the NAT; the signal of this mode, reconstructed by the WMPalgorithm, as well as the signal approximation error are presented here. In this case, the process takes the form of a chirp signal with an increasing time-dependent instantaneous frequency. The wavelet map of the same figure shows the experimentally recorded upward forward transient process when the CT deviates to the right from the NAT, or also the ascending, but reverse process of forced compensation of the left CT deviation, which returns the current trajectory to the NAT. The transient process is displayed in the Wigner wavelet distribution format. Here, in combined form, TFDs of two continuous stationary CTs at frequencies of $2.03 \mathrm{~Hz}$ and $2.50 \mathrm{~Hz}$ are displayed, as well as TFDs of two stationary start-stop CTs with the intermittent mode parameters:

- at a frequency of $0.99 \mathrm{~Hz}$ - the duration of the movement interval is $10 \mathrm{~s}$, the stop is within $8 \mathrm{~s}$, the period of cycle for the UMV movement along a discrete straight trajectory is $18 \mathrm{~s}$. Thus, the driving mode index is $60 \%$;

- at a frequency of $0.82 \mathrm{~Hz}$ - the duration of the movement interval is $8 \mathrm{~s}$, the stop is 7.8 $\mathrm{s}$, the period of the UMV movement cycle along a discrete straight trajectory is $15.8 \mathrm{~s}$. Driving mode index is $50 \%$. 

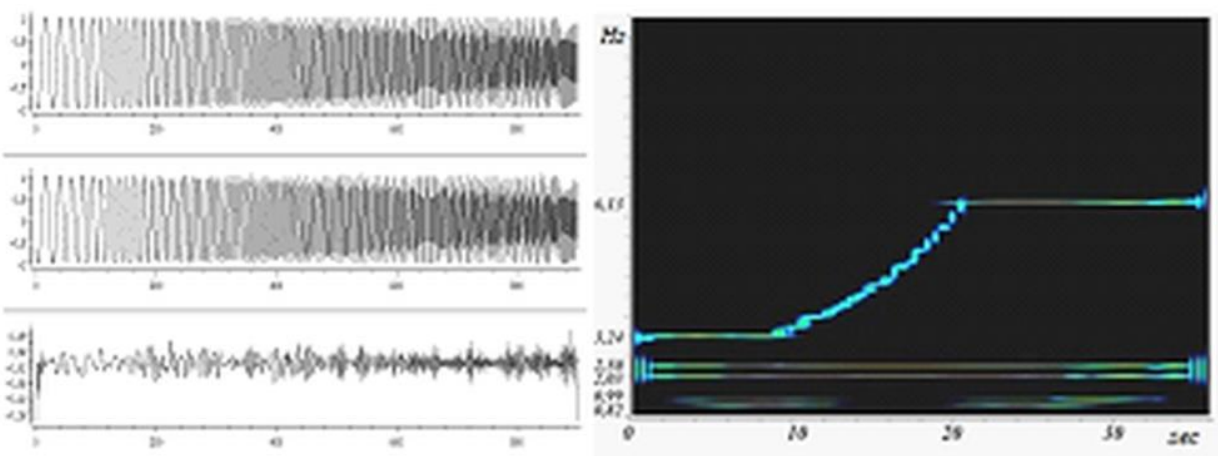

Fig. 3. Left - a one-dimensional signal (in the UMV CT external and autonomous control subsystems) of forming the CT deviating to the right of the NAT

On the right - time-frequency representation of the upward forward transient process (autonomoussporadic) or the reverse process (forced-modal); the chirp signal frequency varies in the range of $\mathrm{f}$ $(\mathrm{t})_{\text {var }}=3.24 \ldots 6.13 \mathrm{~Hz}$

Note that the upward reverse transient process (that is, forced-modal [13, 14]) when implementing the procedure for compensating the CT deviation to the left of the NAT is adopted in the computer-aided control system in the same form as the upward forward process when the CT deviates to the right of the NAT. This is taken up with the purpose of forming the processes that restore a nominal mode of UMV motion in its natural form - in terms of UMV actuators functioning (braking and acceleration).

With continuous monitoring the dynamic state of the UMV CT on a route in the wavelet environment, the procedure of processing a 1D-trajectory signal is followed by a complex operation of constructing the Wigner time-frequency maps, the algorithm of which is shown in Fig. 4. Note that as a result of constructing the CT deviation signal TFD, the ACSS / ECSS subsystem sends a command to the on-board UMV chassis actuators which form the initial («forward») two-exponential section of the deviation CT in the form of a second-order aperiodic transient function $[13,14]$. At the end of the neutralization of a sporadic disturbance caused by the appearance of an obstacle on the way of UMV, the system forms a forced-modal («reverse») transient process that compensates for the CT deviation from the NAT. 


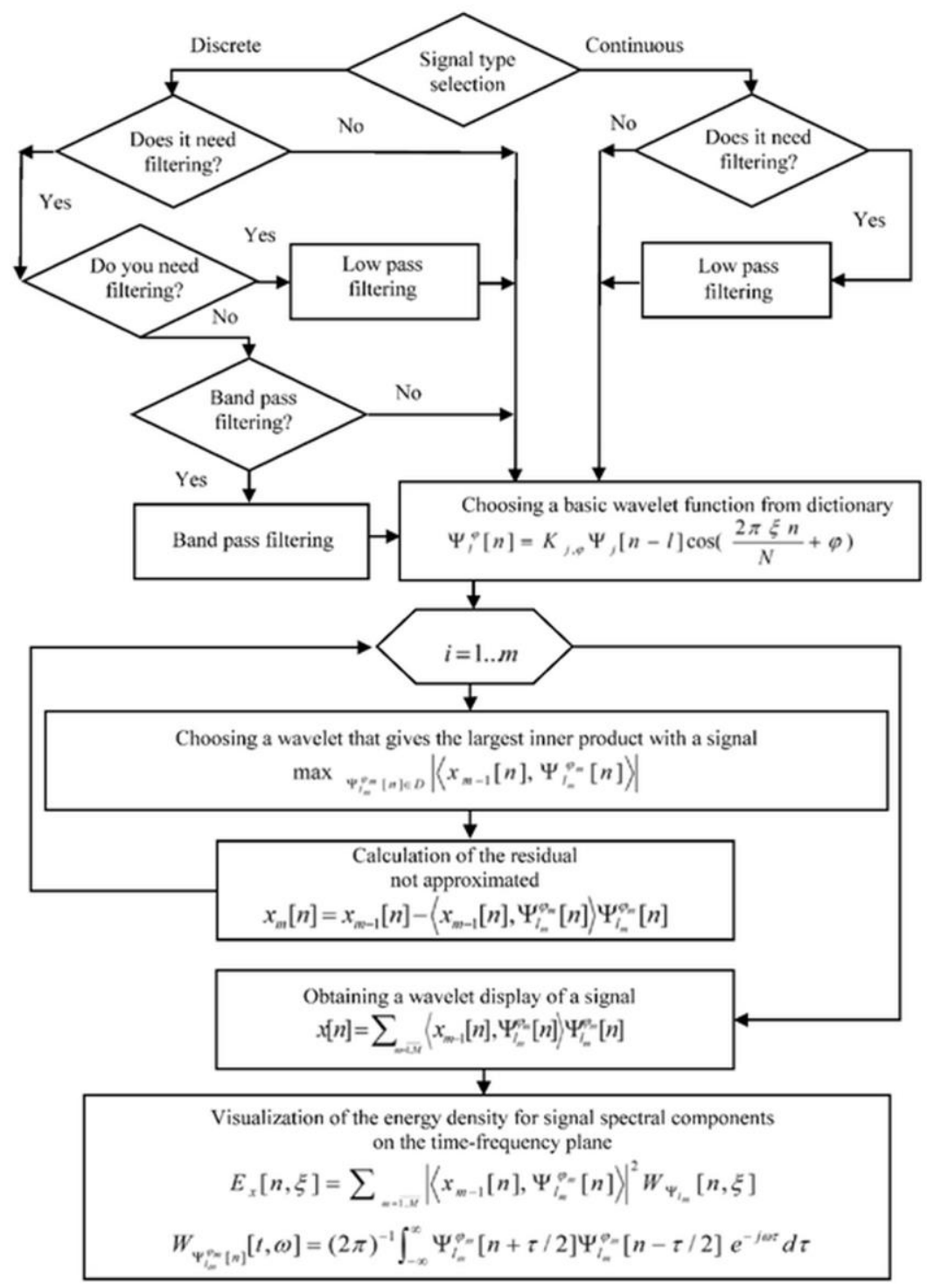

Fig. 4. Algorithm for generating the 3D-representation of the UMV current trajectory onedimensional signal

After performing preliminary procedures related to filtering trajectory signals, a CTsignal is projected onto the dictionary of wavelet functions $[5,4]$ containing Gabor wavelets $[4,6]$ having four parameters: translation $l$, scale $N$, frequency $\xi$, and sinusoidal component initial phase $\varphi$.

Further, in the iterative cycle, at the current iteration number $i=1 \ldots m$, the procedure of choosing from the dictionary a certain wavelet function that satisfies the condition of maximizing the inner product $[4,5,15]$ with a fragment of the approximated CT-signal, is implemented. The next step in computing the wavelet representation of the signal is to calcu- 
late the non-approximated residue that is also approximated at subsequent iterations, and the procedure for projecting the signal onto the dictionary is repeated.

Ultimately, the sum of wavelet functions selected from the dictionary, with their coefficients in the form of inner products of the signal and corresponding wavelets selected from the dictionary is an adaptive approximation $[4,5]$ of the analyzed one-dimensional trajectory signal.

After the end of the process of the adaptive CT-signal approximation with Gabor wavelet functions, a wavelet display of the signal in the form of a wavelet series is formed in the system. Thereafter, the CT-signal 3D-time-frequency distribution proper is visually displayed on the $\mathrm{W}$-map in the form of the signal spectral components energy density $\operatorname{Ex}(n, \xi)$ on the time-frequency plane:

$$
\begin{gathered}
E_{x}[n, \xi]=\sum_{\xi} \sum_{\tau} \sum_{s}\left|\left\langle x(n), \psi_{(s, \tau, \xi)_{m}}(n)\right\rangle\right|^{2} \cdot W_{\psi_{(s, \tau, \xi) m}}(n, \xi)= \\
=\sum_{m=1}^{M}\left|\left\langle x_{m-1}(n), \psi_{(s, \tau, \xi)_{m}}(n)\right\rangle\right|^{2} \cdot W_{\psi_{(s, \tau, \xi) m}}(n, \xi)
\end{gathered}
$$

According to the information presented above, the wavelet functions are selected from the redundant dictionary by the criterion of maximizing the inner product between the signal and a corresponding wavelet. Note that the operation of calculating the inner product is preceded by the procedures for centering, sampling and normalizing an initial signal about the CT dynamic state. In other words, the original signal after centering and sampling is transformed in accordance with the operation of bringing to the unity quadratic norm [4, $15]$.

The wavelet matching pursuit algorithm with the Gabor dictionary provides the most accurate description of time-frequency structures among the currently available methods [4]. This algorithm using the Gabor dictionary has the greatest accuracy in describing timefrequency structures, since it describes components represented in the signal in terms of their time of occurrence, frequency and time coverage, amplitude and phase - with a resolution that can be adjusted to theoretical limits.

\section{Conclusion}

The research results show that the mathematical technique of wavelet transforms is an effective way for computer-aided monitoring and controlling the dynamics of UMV moving along routes within open pit roads.

The paper considers the procedures for converting one-dimensional signals characterizing the dynamic state of UMV CTs on technological routes in an open coal mine into their multidimensional time-frequency representatons. The need for such a transformation of the signal functions of one-dimensional space into a multidimensional wavelet medium makes it possible to monitor the dynamic state of unmanned vehicles in this medium in an information-rich and functionally transparent manner and to control their current route trajectories in a computer-aided mode in open pit mining.

The work is performed with the financial support of the Ministry of Science and Higher Education of the Russian Federation under the agreement No 075-11-2019-034 dated 22.11.2019 with PJSC KAMAZ on the integrated project "Development and creation of high-tech production of autonomous 
heavy platforms for unmanned mining of minerals in the "Smart pit" system, with participation of T.F. Gorbachev Kuzbass State Technical University in terms of research, development and technological works.

\section{References}

1. I.V. Chicherin, B.A. Fedosenkov, I.S. Syrkin, V.Yu Sadovets, D.M. Dubinkin, Mining Journal, 8, 109 (2020)

2. D.M Dubinkin, Mining equipment and electromechanics, 6, 8 (2019)

3. I.V. Chicherin, B.A. Fedosenkov, I. S. Syrkin, V.Y. Sadovets, D.M. Dubinkin, Universities News. Mining Journal, 2, 103 (2021)

4. S. Mallat, A wavelet tour of signal processing ( Academic Press, New York, Ecole Politechnique, Paris, Courant Institute, New York, 1999; Reprinted, 2001, 3d Edition. The Sparse Way. Academic Press, 2008)

5. S. Mallat, Z. Zhang, IEEE Transactions on Signal Processing, 12, 3397 (1993)

6. J.C. Goswami, A.K. Chan, Fundamentals of Wavelets: Theory, Algorithms and Applications( A Wiley Interscience Publ., John Wiley \& Sons, Inc., New York,1999)

7. F. Auger, E. Chassande-Mottin, ISTE, January 131 (2008).

8. D.B. Fedosenkov, A.A. Simikova, I.V. Sudakov, B.A. Fedosenkov, CAD / CAM / PDM - 2016. 241 (2016)

9. B.Boashash, Measures, performance assessment, and enhancement TFDs, January $\mathbf{3 8 7}$ (2016)

10. D.B. Fedosenkov, Steel in Translation, 49, 4, 252 (2019)

11. L. Debnath, PINSA, January 35 (2002)

12. L. Debnath, Wavelet transforms and their applications (Birkhauser, Boston, 2002)

13. R.S. Dorf, R. H., Bishop Modern control systems (Laboratory of Basic Knowledge, Moscow, 2002)

14. Goodwin, C. Graham Control System Design (Pearson Education, Inc., Prentice Hall, New York, 2001)

15. G.A. Korn, T.M. Korn, Mathematical Handbook for Scientists and Engineers: Definitions, Theorems, and Formulas for Reference and Review(Dover Civil and Mechanical Engineering, New York, 2000) 